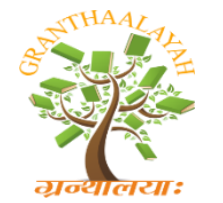

$$
\begin{gathered}
\text { INTERNATIONAL JOURNAL OF RESEARCH - } \\
\text { GRANTHAALAYAH } \\
\text { A knowledge Repository }
\end{gathered}
$$

RACSIT - 17

\title{
EARLY DIAGNOSIS OF SKIN CANCER USING ARTIFICIAL NEURAL NETWORKS
}

\author{
Birajdar Yogesh ${ }^{* 1}$, Rengaprabhu $\mathbf{P}^{2}$ \\ ${ }^{*} 1,2$ Department of electronics and communication, Don Bosco Institute Of Technology, \\ Bangalore, India
}

DOI: https://doi.org/10.29121/granthaalayah.v5.i4RACSIT.2017.3341

\begin{abstract}
The proposed work is to present an approach to easily detect the skin cancer and classify into benign and malignant classes differentiating with the wounds. The skin cancer occurs for many people in some regions of the countries like Australia \& New Zealand where the sunlight is difficult to reach during winters. Thus the deficiency of Vitamin D causes skin cancer for the people dwelling in such regions. Self-assessment is being encouraged in such cities to detect the skin cancers in early stages. It is very much essential to diagnose the skin cancer in the early stages and help the patients to get the treatment effectively. The proposed system is supposed to match the self-assessment need for the people to take care of themselves in very less time and regular basis.
\end{abstract}

Keywords: Classification; Feature Selection; Image Processing; Snake Segmentation; Support Vector Machine.

Cite This Article: Birajdar Yogesh, and Rengaprabhu P. (2017). "EARLY DIAGNOSIS OF SKIN CANCER USING ARTIFICIAL NEURAL NETWORKS." International Journal of Research - Granthaalayah, 5(4) RACSIT, 1-7.

\section{Introduction}

Recently, skin lesion recognition has drawn great attention and has become a popular research topic. In 2012, multiple types of skin cancer, including basal cell carcinoma, squamous cell carcinoma and malignant melanoma, occurred in 231,000 people and caused 55,000 deaths with highest rates in New Zealand and Australia. Historical data indicated that skin cancer has been commoner in the last 20 years in areas that are mostly Caucasian. Skin cancer could spread to other organs in the body. It begins by changing the skin cell, which is formed by Melanocytes, a pigment-containing cancer cell in the skin.

The Skin Cancer Foundation recommends that everyone practice monthly head-to-toe selfexamination of their skin, so that they can find any new or changing lesions that might be 
cancerous or precancerous. Skin cancers found and removed early are almost always curable. Learn about the warnings signs of skin cancer and what to look for during self-examination. If you spot anything suspicious, see a doctor.

There are three main types of skin cancer:

- Basal cell carcinoma,

- Squamous cell carcinoma, and

- Melanoma.

\section{Related Work}

The presented alternative processes for melanoma identification via dermoscopy imagery, based on surface texture and shade of colour feature extraction. Texture and colour were compared in performance in their work developed a portable classifying scheme and application for epidermal lesion classification. Their work is composed of image segmentation, feature extraction and classification. Empirical testing of this system presented an accuracy of $67 \%$ for malignant classification. Early diagnosis of melanoma is crucial and could lead to successful treatment. In real-life, a well-trained dermatologist could reach approximately 80\% accuracy of diagnoses through visual inspection with specificity and sensitivity. Such diagnosis is conducted based on the ABCDE (Asymmetry, Border, Colour, Diameter and Enlargement) guidelines.

The Adaptive Snake (AS) approach is chosen because it is efficient for establishing a discriminating analysis that divides the image into two classes of pixels. To extract multiple features, such as colour and area, the segmentation results of this threshold-based method are plotted into multiple images.

\section{Proposed System}

The proposed work is to analyze the best and accurate segmentation algorithm for extraction of skin cancer region to detect skin cancer in early stages.

The proposed system has the following steps involved-

- Preprocessing

- Segmentation

- Feature extraction

- Classification 
Machine Learning Procedure

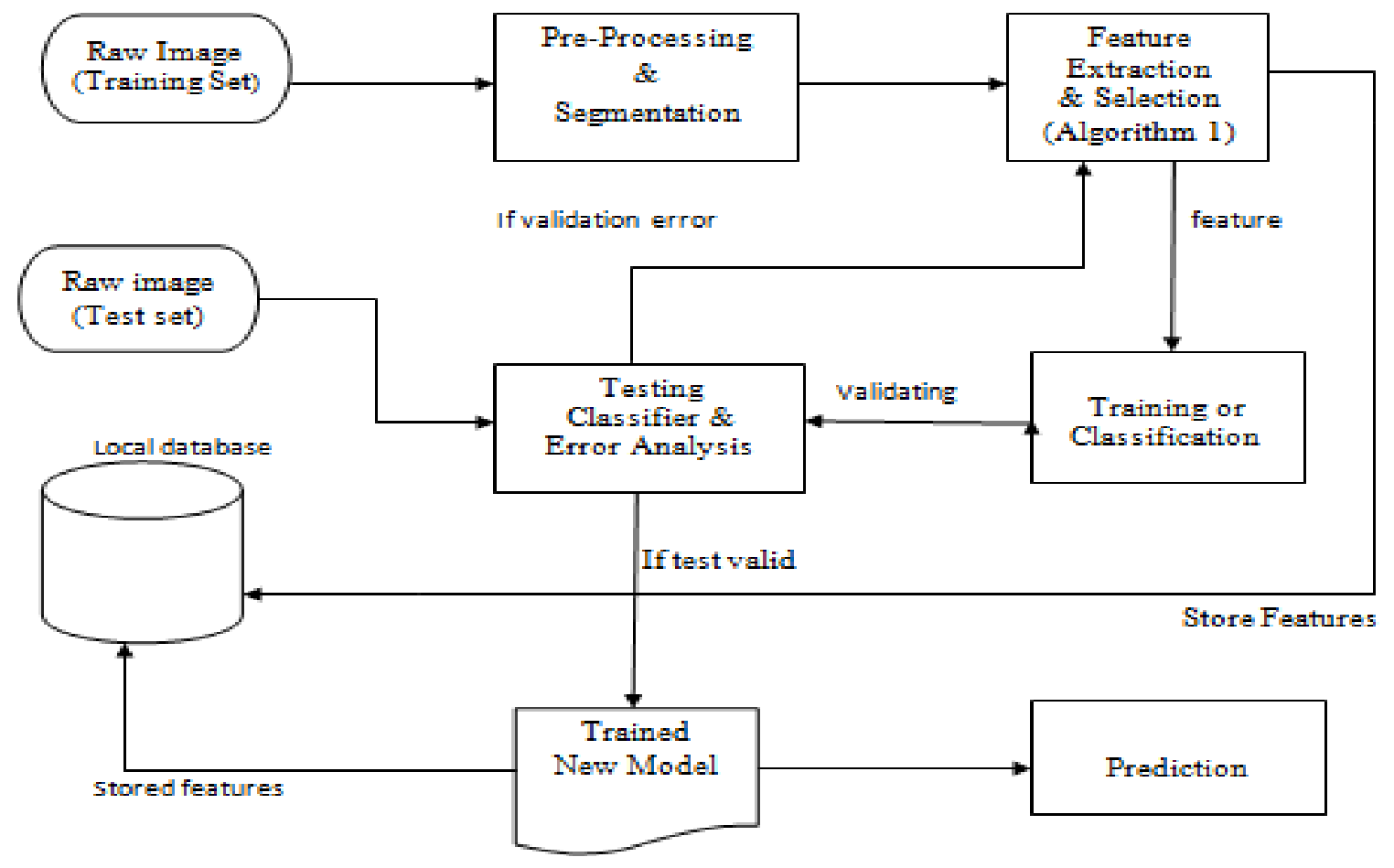

Figure1: The system architecture of Cancer Diagnosis System

Pre-Processing: A pre-processing method is essential for benign and malignant skin lesions classification. This involves transforming raw data into an understandable format for further processing. In the real world, data are often inconsistent and incomplete and may contain many errors. Removing any noise and unnecessary features which cause confusion to classifiers is required.

1) Hair Remover: The Enhanced Dull Razor algorithm was used to remove hairs from images where morphological closing image processing was generalised to grey-level images, followed by identification of the narrow, elongated hair outline. Bilinear interpolation was implemented to substitute the identified pixels of the hairs. Mat lab 'roifill' function was used to perform a fill operation using an interpolation method based on Laplace's equation. This step resulted in a smooth fill inward from the borders of the region of interest.

2) Contrast Enhancement: Subsequently, the image clarity was enhanced by improving the shape and edges of the image. Image borders were sharpened using contrast enhancement. This process may also optimise subsequent segmentation accuracy.

3) Gray scale Conversion: RGB images of lesions, with $360 \times 360$ pixels in size, were transformed to greyscale by removing hue and saturation using a process which computes the weighted sum of the colour components.

4) Noise Filtering: Median filtering was used to effectively remove 'salt and pepper' noise, thus diminishing the effect of small objects such as thin hairs and outlying regions of pixels caused, for example, by air bubbles. 
Segmentation: Image segmentation is a technique to determine the shape and size of the border, and to separate the object from its background based on different features extracted from the image. After removing the noise from the lesion area, the lesion needs to be separated from the skin, and therefore the analysis for diagnosis is conducted purely using the necessary area.

The Adaptive Snake (AS) approach is chosen because it is efficient for establishing a discriminating analysis that divides the image into two classes of pixels. To extract multiple features, such as colour and area, the segmentation results of this threshold-based method are plotted into multiple images.

Feature Extraction: After segmentation, image features are extracted for the subsequent classification. Several methods have been identified for feature extraction. Overall, the majority of related work employed the ABCDE rules of dermatology for feature extraction. In this research, measurements such as compactness index, fractal index, and edge abruptness are used in order to indicate border irregularity.

Table 1: Features Extracted from Lesion Image

\begin{tabular}{|c|c|c|}
\hline Features & Feature list & Feature type \\
\hline F1 & Border asymmetry index & \\
\hline $\mathrm{F} 2$ & Perimeter & \\
\hline F3 & Extent & \\
\hline F4 & Equivalent diameter & \\
\hline F5 & Asymmetry index & \\
\hline F6 & Form factor & shape \\
\hline F7 & Roundness & \\
\hline F8 & Major and minor difference & \\
\hline F9-f10 & $\begin{array}{l}\text { Compactness index } 1 \\
\text { Compactness index } 2\end{array}$ & \\
\hline F11-f12 & $\begin{array}{l}\text { Relative chromacity } \\
\text { Color ratio }\end{array}$ & Color \\
\hline F13-15 & \begin{tabular}{l}
\multicolumn{2}{l}{ Colour Mean } \\
Colour Standard Colour \\
(RGB, HSV, Lab) \\
Ration mean colour
\end{tabular} & $\begin{array}{l}\text { Colour Standard } \\
\text { (RGB, HSV, Lab) }\end{array}$ \\
\hline
\end{tabular}

\section{Shape}

a) Asymmetry: A melanocyte lesion may be diagnosed by a number of identifiers, of which one of the most significant is a lack of symmetrical morphology. In dermatology terms the $\mathrm{ABCDE}$ rule model rates this aspect as the most crucial factor. In consideration of the symmetry feature, a number of factors are concurrently relevant, including colour, texture and morphology. A three-fold classification system can be derived from measuring symmetry, with three-class out puts representing total symmetry, a lack of symmetry along a single axis and a lack of symmetry along dual axe, respectively. The lesion asymmetry was evaluated by calculating the area with inner and outer of the lesion, using the formula shown as follows. 


$$
\mathrm{AI}=\frac{\Delta A K}{A L} * 100
$$

Where, $A I$ represents asymmetry Index. $\triangle A K$ represents the area between the two halves of the lesion and $A L$ denotes the lesion area.

b) Border Irregularity: Irregularities occurring in the edge of a malignant lesion offer useful information concerning that lesion's nature. Typically, the edge of a malignant lesion usually exhibits four factors of interest, i.e. density, fractal dimension, radial variability and the extent to which its contour exhibits small irregularities. To identify the lesion border irregularity.

$$
\mathrm{I}=\frac{\mathrm{ab}}{2 \pi(a 2+b 2)} \frac{p 2}{\Delta A}
$$

Where, I represent irregularity with $a$ and $b$ representing the lengths of major and minor axes of the lesions. $P$ represents the perimeter of the lesion and $\Delta A$ indicates the area of corresponding.

c) Compactness: Another relevant feature is the degree to which the lesion can be described as compact. In order to determine this aspect, a comparative analysis is performed between the lesion's boundary and a circle with a circumference of the same length. It is the former of these two numerical values that presents a challenge in its assessment.

$$
C=\frac{4 \pi p 2}{\Delta A}
$$

Where, C represents the compactness.

d) Classification: Selected features are used for the recognition and classification of benign and malignant lesions. A wide range of classifiers is explored. Eventually, the SVM classifier was used for benign and malignant skin lesion classification because of its superior performances. SVM is a supervised learning method to solve regression and classification problems. SVM is performed by creating the n-dimensional hyperplane that separates data into two classes. The goal of SVM is to pinpoint an optimum hyperplane which can separate clusters from vectors to categorise the target variables on one side and the other class on the other side. The support vectors refer to the vectors near the hyperplane.

\section{Experiments and Discussions}

In this research, we employ 1200 images for training and 100 images for testing from the above database. Although the AS is comparatively superior in its delivered outcomes, the experiments also revealed shortcomings, due to the difficulty of global maxima finding, the existence of which will be the subject of further research. The GA approach has been applied in this research to select relevant and reliable factors and carry out skin lesion categorization. The results of the experiments were compared with those obtained for related research such as $[10,26]$. The following formula was used to calculate accuracy, sensitivity and specificity for system evaluation. 


$$
\begin{aligned}
& \text { Accuracy }=\frac{\text { total of detected lesions }}{\text { total dataset images }} * 100 \\
& \text { Sensitivity }=\frac{T P}{T P+F N} \\
& \text { Specificity }=\frac{T N}{T N+F N}
\end{aligned}
$$

In this research, we employ $80 \%$ images for training and $20 \%$ images for testing from the above database. Although the AS is comparatively superior in its delivered outcomes, the experiments also revealed shortcomings, due to the difficulty of global maxima finding, the existence of which will be the subject of further research. The AS approach has been applied in this research to select relevant and reliable factors and carry out skin lesion categorization.

The results show that our model obtains promising performance with an average of $92 \%$ and $84 \%$ accuracy rates for benign and malignant cases.

\section{Conclusions}

This research proposes an intelligent decision support system for the identification of benign and malignant skin lesions from dermoscopic images, which offers the prospect of achieving improved and more accurate classification of lesions from images. The proposed system employs pre-processing such as dull razors and median filters to remove hair and other noise. Then, the images were segmented using a pixel limitation technique to separate lesions from image background. Feature extraction is subsequently conducted. The features extracted by our system reflect the well-known asymmetry, border irregularity, colour variegation and diameter (ABCD) of dermatology. Our work achieves an average accuracy of $92 \%$ and $84 \%$ for benign and malignant skin lesion classification.

\section{References}

[1] R. O. Duda, P. E. Hart, and D. G. Stork. Pattern Classification. John Wiley, New York, 2001.

[2] Y.W. Lim and S.U. Lee, "On the color image segmentation algorithm based on the thresholding and the fuzzy C-means techniques, "Pattern Recognition, vol. 23, no.9, pp. 935-952, 1990.

[3] M.M. Chang, M.I. Suzan, and A. M. Tekalp, "Adaptive Bayesian estimation of color images,"J Electron. Imaging, vol. 3, pp. 404-414, October 1994.

[4] Canny, J. F. (1986). A computation approach to edge detectors. IEEE Transactions on Pattern Analysis and Machine Intelligence, 8, 34-43.

[5] Gomez-Moreno, H., Maldonado-Bascon, S., \& Lopez-Ferreras, F.(2001). Edge detection in noisy images using the support vector machines. IWANN (1) (pp. 685-692).

[6] Lehmann, F. "Turbo segmentation of textured images", on Pattern Analysis and Machine Intelligence, vol: 33,pp: 16 - 29,2011

[7] Sboner, A., Eccher, C., Blanzieri, E., Bauer, P., Cristofolini, M., Zumiani, G. \& Forti, S. 2003. A multiple classifier system for early melanoma diagnosis. Artificial Intelligence in Medicine, 27, $29-44$.

[8] Skin Cancel Foundation. No Date. Available: http://www.skincancer.org/skin-cancerinformation/ melanoma/melanoma-warning-signs-and-images/do-youknow-your-abcdes\#panel1-1 [Accessed 24 December 2014]. 
[9] Schmid, P. 1999. Segmentation of digitized dermatoscopic images by two-dimensional color clustering. Medical Imaging, IEEE Transactions on, 18, 164-171.

[10] Gilmore, S., Hofmann-Wellenhof, R. \& Soyer, H. P. 2010. A support vector machine for decision support in melanoma recognition. Experimental dermatology, 19, 830-835.

[11] Ramlakhan, K. \& Shang, Y. A Mobile Automated Skin Lesion Classification System. 2011 IEEE 23rd International Conference on Tools with Artificial Intelligence, 7-9 Nov. 2011 2011. 138141.

[12] Barata, C., Ruela, M., Francisco, M., Mendonça, T. \& Marques, J. S. 2014. Two systems for the detection of melanomas in dermoscopy images using texture and color features. Systems Journal, IEEE, 8, 965-979.

[13] Neoh, S.C., Srisukkham, W., Zhang, L, Todryk, S., Greystoke, B., Lim, C.P., Hossain, A. And Aslam, N. 2015 An Intelligent Decision Support System for Leukaemia Diagnosis using Microscopic Blood Images. Scientific Reports, 5 (14938). Nature Publishing Group.

[14] Zhang, L., Jiang, M., Farid, D. And Hossain, A.M. (2013). Intelligent Facial Emotion Recognition and Semantic-based Topic Detection for a Humanoid Robot. Expert Systems with Applications, 40 (2013), 5160-5168.

[15] JF Khan, SMA Bhuiyan ,'Image Segmentation and Shape Analysis for Road-Sign Detection"on Intelligent Transportation Systems, Vol:12,pp: 83-96, 2011

[16] D Krstinic, AK Skelin, I Slapnicar,'Fast two-step histogram-based image segmentation", on, IET, 2011

[17] Felzenszwalb, P.F., Huttenlocher, D.P.: Efficient graph-based image segmentation', Int. J. Comput. Vis., 2004, 59, (2), pp. 167-181

[18] Mushrif, M.M., Ray, A.K.: 'Color image segmentation: Rough set theoretic approach', Pattern Recognit. Lett., 2008, 29, (4), pp. 483-493

[19] Chen, T.Q., Lu, Y.: 'Color image segmentation: an innovative approach', Pattern Recognit., 2002, 35, (2), pp. 395-405

[20] Yu, Z., Wong, H.: 'A rule based technique for extraction of visual attention regions based on realtime clustering', IEEE Trans. Multimedia, 2007, 9, (4), pp. 766-784

[21] Y. B. Chen and O. T. -C. Chen, "Image segmentation method using thresholds automatically determined from picture contents," EURASIP Journal on Image and Video Processing, Article ID140492, 2009, doi:10.1155/2009/140492.

[22] Kurugollu, F., Sankur, B., Harmanci, A.E.: 'Color image segmentation using histogram multi thresholding and fusion', Image Vis. Comput., 2001, 19, (13), pp. 915-928

[23] Baradez, M.O., McGuckin, C.P., Forraz, N., Pettengell, R., Hoppe, A.: 'Robust and automated unimodal histogram thresholding and potential applications', Pattern Recognit., 2004, 37, (6), pp. 1131-1148

[24] Fan, J., Zeng, G., Body, M., Hacid, M.: 'Seeded region growing: an extensive and comparative study', Pattern Recognit. Lett., 2005, 26, (8), pp. 1139-1156

*Corresponding author.

E-mail address: ybirajdar543@gmail.com 